\title{
Increased blood-cerebrospinal fluid transfer of albumin in advanced Parkinson's disease
}

\author{
Valerio Pisani ${ }^{1}$, Alessandro Stefani ${ }^{1}$, Mariangela Pierantozzi ${ }^{1}$, Silvia Natoli ${ }^{2}$, Paolo Stanzione ${ }^{1}$, Diego Franciotta ${ }^{3}$ \\ and Antonio Pisani $i^{1,4^{*}}$
}

\begin{abstract}
Background: Alterations in blood-brain barrier permeability have been proposed to represent a relevant factor contributing to Parkinson's disease progression. However, few studies have addressed this issue in patients at different stages of disease.

Methods: Albumin was measured in cerebrospinal fluid and serum samples obtained from 73 non-demented subjects with idiopathic Parkinson's disease and 47 age-matched control subjects. The albumin ratio (AR) was calculated to assess blood-cerebrospinal fluid and blood-brain barrier function. The group of patients with Parkinson's disease included 46 subjects with Hoehn-Yahr staging between 1 and 2 and 27, with a score ranging from 2.5 to 4.

Results: Statistically significant differences in albumin ratio were found between patients with advanced disease, and both early-stage and unaffected groups. Conversely, early-phase patients did not differ from healthy subjects. Additionally, dopaminergic treatment seems to exert a possible effect on AR values.

Conclusions: Our study demonstrates that possible dysfunction of the blood-cerebrospinal fluid barrier, blood-brain barrier, or both, characterize Parkinson's disease progression. The associations between clinical scores, treatments and biochemical findings suggest a progressive impairment of barrier integrity during the course of the disease.
\end{abstract}

Keywords: Albumin ratio, Blood-brain barrier, Blood-cerebrospinal fluid barrier, Cerebrospinal fluid, Parkinson's disease

\section{Background}

A growing body of evidence suggests an important role of inflammation in the pathophysiology of neurodegenerative diseases. Moreover, an impairment of brain barrier filtering systems through direct (leukocyte infiltration, toxins) or indirect (cytokines, growth factors) pathophysiological mechanisms has been proposed in Parkinson's disease (PD) $[1,2]$. Particularly, a positron emission tomography (PET) study in PD patients revealed a dysfunction of the blood-brain barrier (BBB) transporter system [3], and increased BBB permeability has also been demonstrated in rat models of PD [4,5]. However, a detailed analysis on inflammation-induced changes of barriers that separate

\footnotetext{
* Correspondence: pisani@uniroma2.it

'Department of Neuroscience, University of Rome 'Tor Vergata', Rome, Italy ${ }^{4}$ Laboratory of Neurophysiology, Santa Lucia Foundation, IRCCS, Rome, Italy Full list of author information is available at the end of the article
}

blood from cerebrospinal fluid (CSF) and brain parenchyma in PD is still lacking.

Choroid plexuses, namely the blood-CSF barrier (BCSFB), produce two-thirds of the CSF volume, the remaining one-third deriving from the $\mathrm{BBB}$ [6]. The analysis of protein content on lumbar CSF specimens mainly allows the assessment of the functional integrity of the BCSFB, but not the isolated BBB $[7,8]$. Thus, in attempt to gain a better understanding on the role of BCSFB and BBB function on disease course, we analyzed paired CSF and serum samples and calculated albumin ratio (AR) of PD patients at different disease stages, including both early de novo subjects and clinically advanced ones.

\section{Methods}

We screened 102 consecutively admitted and unselected subjects with idiopathic PD according to the UK PD

\section{Biomed Central}


Society Brain Bank criteria [9]. Patients underwent a thorough clinical and neurological evaluation comprehensive of severity assessment (Unified PD Rating Scale (UPDRS) part III and Hoehn-Yahr staging (H\&Y)), brain magnetic resonance imaging (MRI), and atraumatic lumbar puncture for CSF analysis. CSF and blood samples were also obtained for diagnostic purposes from 47 agematched control subjects (CTRL), hospitalized because of complaining non-specific pain, motor or sensory symptoms, though not presenting medical conditions known to impair BCSFB and BBB permeability (Table 1). In order to avoid any potential bias, we also added a second independent cohort of 11 subjects (IND) complaining a pharmacoresistant headache, without any evidence for subarachnoid hemorrhage or cerebral neoplasm. Exclusion criteria were: Mini Mental State Examination (MMSE) score <26, evidence of large cortico-subcortical lesions or confluent cerebral infarctions on MRI scans, subjects exhibiting low-back pain or radiculopathies, abnormal CSF cell count $(>4$ cells $/ \mu \mathrm{l})$, and the presence of intrathecal IgG synthesis, whether quantitatively or qualitatively assessed [8], on routine CSF analysis. All subjects underwent lumbar puncture in the morning of the same day of the clinical evaluation. Patients were punctured lying in lateral position with atraumatic needles. CSF was collected in polypropylene tubes using standard sterile techniques. The first sample $(2 \mathrm{ml})$ was utilized for routine analysis, while a second sample $(5 \mathrm{ml})$ was collected to measure albumin-IgG concentration and oligoclonal bands. Blood specimens were also obtained at the same time of lumbar procedure. Immediately after collection, CSF samples were stored immediately on ice, sent to the local laboratory, and processed within $1 \mathrm{~h}$. Albumin and IgG concentrations were determined by immunonephelometry and oligoclonal bands by isoelectric focusing method. Then, albumin ratio (CSF albumin/serum albumin concentration $\times 10^{-3}$ ), and IgG ratio (CSF IgG/serum IgG concentration $\times 10^{-3}$ ), as additional measures of BCSFB and BBB permeability, were calculated [7]. PD patients were then divided into two groups according to H\&Y staging, considering 2 as the grouping cut-off score to compare early with advanced PD subjects [10]. All procedures were carried out with the appropriate understanding and written consent of the subjects. The research protocol had been previously approved by the Ethical Committee of Policlinico Tor Vergata Foundation.

\section{Statistical analysis}

Data are reported as mean, standard deviation and medians (Table 1). Biochemical data, given their non-normal distribution, were separately assessed by one-factor nonparametric Kruskal-Wallis analysis of variance (ANOVA) for four groups. In case of significance, Mann-Whitney test was used to perform multiple comparisons; a post hoc Bonferroni correction was applied considering $P<0.016$ as statistically significant. The $\chi^{2}$ test or Fisher exact test (if there were less than five observations) were used for frequency data, in order to rule out the possibility that differences among groups could depend on random causality. $P$ values less than 0.05 were considered significant. Analysis was performed using SigmaStat 3.5 software (Systat Software, Inc., Point Richmond, CA, USA).

Table 1 Clinical data and cerebrospinal fluid (CSF) findings in Parkinson's disease (PD) patients and controls

\begin{tabular}{lllll}
\hline & CTRL & IND & PD 1-2 & PD 2.5-4 \\
\hline No. of patients & 47 & 11 & 46 & 27 \\
Age in years & $60.4 \pm 10.9(45.0$ to 83.0$)$ & $59.2 \pm 9.0(45.0$ to 75.0$)$ & $60.3 \pm 9.2(44.0$ to 77.0$)$ & $63.9 \pm 10.1(49.0$ to 83.0$)$ \\
Disease duration, months & - & - & $25.8 \pm 15.1$ & $54.0 \pm 41.7$ \\
UPDRS III score & - & - & $19.0 \pm 5.6$ & $38.0 \pm 10.6$ \\
H\&Y stage & - & - & $1.6 \pm 0.4$ & $3.0 \pm 0.5$ \\
PD medications & - & - & None: $65.2 \%$ & None: $7.4 \%$ \\
& & DA: $19.6 \%$ & DA: $22.2 \%$ \\
& & LD: $4.3 \%$ & LD: $33.3 \%$ \\
Motor fluctuations & - & LD +DA: $10.9 \%$ & LD + DA: $37.1 \%$ \\
Albumin ratio $\left(\times 10^{-3}\right)$ & $5.2(2.3$ to 13.1$)$ & - & $7 / 46$ & $17 / 27$ \\
IgG ratio $\left(\times 10^{-3}\right)$ & $2.7(1.1$ to 6.9$)$ & $5.8(3.4$ to 8.1$)$ & $5.6(2.6$ to 16.2$)$ & $8.2(3.0$ to 21.2$)$ \\
\hline
\end{tabular}

The top half of the table shows clinical data expressed as mean \pm SD (minimum to maximum). The lower half shows biochemical values expressed as median (minimum to maximum).

$C T R L=$ control group of healthy subjects; $D A=$ dopamine agonists; $H \& Y=$ Hoehn-Yahr; IND = independent group with no neurological disease; $L D=$ levodopa; PD 1-2 = Parkinson's disease patients with H\&Y stage ranging from 1 to 2; PD 2.5-4 = Parkinson's disease patients with H\&Y stage ranging from 2.5 to 4; UPDRS = Unified Parkinson's Disease Rating Scale. 


\section{Results}

Among 102 PD patients screened, 29 did not meet eligible criteria. Specifically, 11 subjects were excluded because of low MMSE scores, 8 reported intense low-back pain and 10 had confluent cerebral infarctions on MRI. Thus, we analyzed CSF/serum data from 73 PD patients, in comparison with control subjects. We found a significant difference in CSF/serum albumin ratio $(P=0.02)$ between the two groups. According to H\&Y staging [10], PD patients (Table 1) were then divided into 2 groups: 46 patients with staging between 1 and 2 (PD 1-2) and 27 subjects ranging between 2.5 to $4 \mathrm{H} \& \mathrm{Y}$ staging (PD 2.5-4). PD 1-2 group showed a mean disease duration of $25.8 \pm 15.1$ months and a mean UPDRS III score of $19.0 \pm 5.6$, whereas PD 2.5-4 group had a mean disease duration of $54.0 \pm 41.7$ months and a mean UPDRS III score of 38.0 \pm 10.6 . The mean ages of PD 1-2 and 2.5-4 patients (Table 1$)$ did not significantly differ $(P=0.13)$.

Albumin $(P=0.002)$ and IgG $(P=0.008)$ ratios were significantly different in the examined groups. PD 2.5-4 patients differed from CTRL, IND and PD 1-2 groups both in albumin $(P<0.001, P<0.01$ and $P=0.002$, respectively) and $\operatorname{IgG}$ ratio values $(P=0.002, P<0.01$ and $P=0.005$, respectively). Conversely, no statistically significant difference $(P>0.05)$ between PD $1-2$ and CTRL, IND groups was found (Figure 1).

Then, we identified the normal cut-off value for AR $\left(8.3 \times 10^{-3}\right)$, which corresponds to the 90th percentile of AR values in the CTRL group. The number of PD 2.5-4 patients with $\mathrm{AR}>8.3 \times 10^{-3}$ was higher than that of $\mathrm{PD}$ $1-2$ patients $(P=0.015)$, thus confirming that the difference between PD groups is not due to random causality. A possible effect of dopaminergic therapy on AR values was assessed by comparing patients with and without pharmacological treatment. The number of treated patients with AR $>8.3 \times 10^{-3}$ was higher than that of untreated patients $(P=0.035)$.

\section{Discussion}

The robust effort in search for biomarkers that might help monitoring degenerative processes has led to a renewed interest and application of lumbar puncture for research purposes in a variety of neurodegenerative diseases. CSF concentrations of protein produced within the CNS, such tau protein or $\alpha$-synuclein, which is also abundantly expressed in red blood cells [11], do not depend on CNS barrier permeability [8]. However, measurement of promising markers of neurodegeneration could be affected by integrity of $\mathrm{BBB}$ and possible dysfunctions of BCSFB. This issue is of relevance especially considering that changes in BBB permeability may occur in advanced PD [1,3], and changes have also been demonstrated in PD animal models [4,5]. Although the main cause of PD remains still unclear, several reports

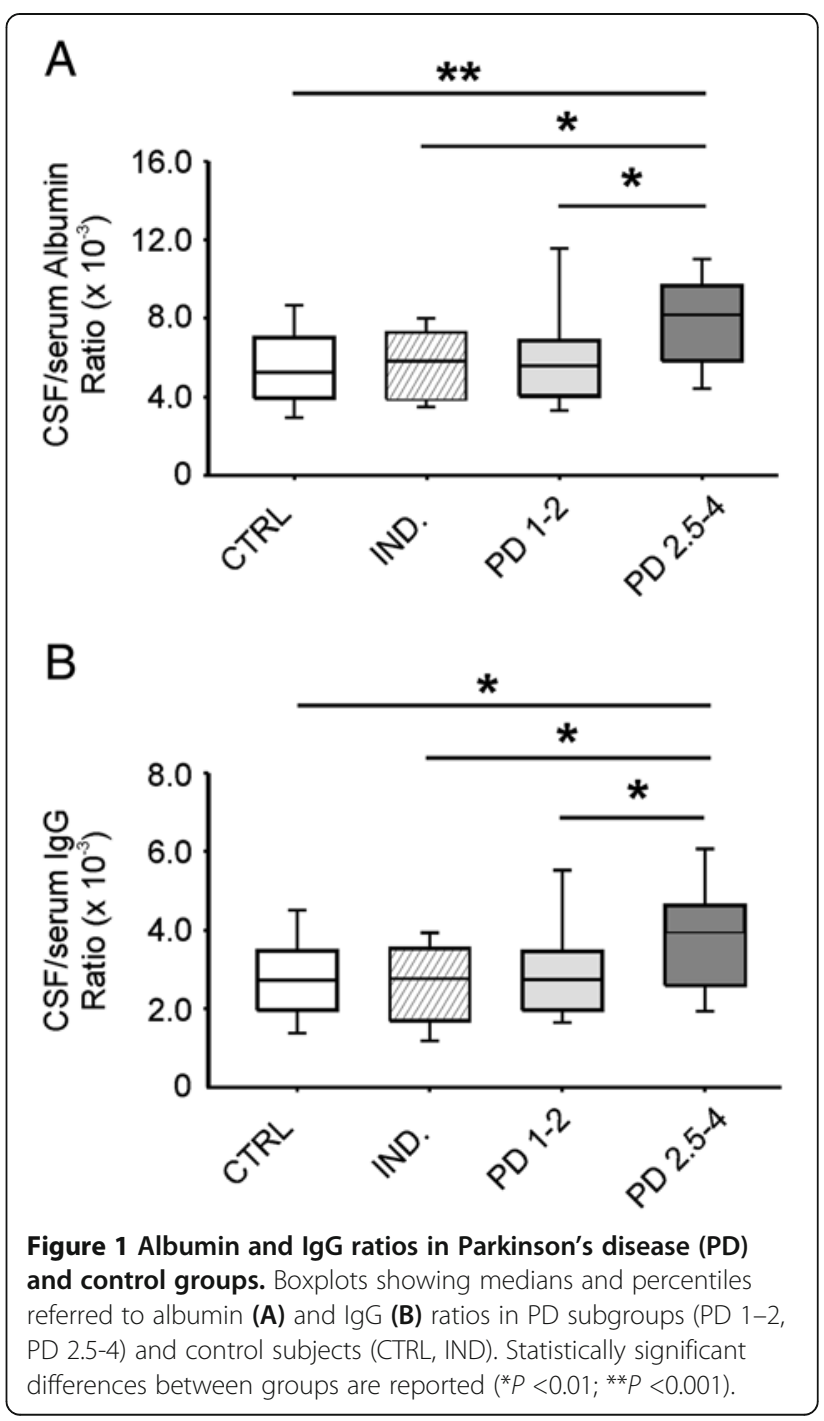

suggest that microglial activation, reactive astrocytes, peripheral immune cells infiltration may be implicated in the development of the disease $[2,12,13]$. Moreover, an increase of brain barrier permeability could allow other elements such as complement, toxins, and metals, normally excluded from the central compartment, to bypass BBB and potentially contribute to the progression of disease. Notably, a BBB dysfunction would likely contribute to alter also ion balance, disrupt transport system, for example, P-glycoprotein $[3,14]$ or $\mathrm{Na}^{+}$-dependent levodopa pump activity [15], and potentially impair enzymatic constituents of the barrier.

In our study, we found AR values to be within the normal range in early-staging PD patients, in accordance with previous data [16], whereas they were abnormally high in more advanced phases of the disease. AR is currently recognized as the most reliable marker of BCSFB permeability $[7,8]$, but the BBB also contributes partially to CSF volume and composition [6]. Therefore, an increase of 
high molecular proteins in the CSF not only indicates BCSFB dysfunction but may also represent an index for BBB impairment. A hypothetical inflammation-dependent and locoregional increase in BBB permeability or a decrease of CSF flow rate by a failure of choroidal Na-K ATPase [17] might explain the increase of blood-derived proteins in the CSF. BBB dysfunction, which is likely sustained by neuroinflammation [2-5], BCSFB hypofunction and decreased CSF flow rate [18], could be meaningful phenomena occurring in advanced stages of PD. However, it is questionable whether such abnormalities play a role as trigger events, participate in the pathogenic process, or are simply consequences of degeneration. Of note, in neurodegenerative diseases, a barrier compromise might also have significant implications for drug delivery, even if BBB alterations do not develop throughout an affected structure in which neurodegeneration occurs, but rather in small localized areas within the structure [5]. Motor complications, such as dyskinesias, that become manifest in advanced stages of the disease after chronic levodopa treatment, could be related to inhomogeneous drug delivery as a result of barrier compromise, or to a reduced capacity of the $\mathrm{Na}^{+}$dependent transport system to remove levodopa from the brain extracellular fluid. The latter hypothesis well fits with a previous study on high levodopa availability in advanced PD patients [19].

\section{Conclusions}

In summary, future neuroimaging studies, along with analyses of proteins that are differently concentrated in the CSF on the basis of the CSF flow rate [8], will clarify the open issues generated by the present study. More insights into the function of CNS barriers could allow the identification of subsets of patients with different responses to drugs, leading to better-tailored therapies.

\section{Competing interests}

The authors declare that they have no competing interests.

\begin{abstract}
Authors' contributions
VP, DF and AP designed the study and wrote the paper. VP and MP enrolled the patients and performed clinical assessment. SN collected CSF samples for biochemical analysis and revised the manuscript. VP and SN carried out offline CSF data analysis and performed biostatistical comparisons. AS and DF gave their advice about the recruitment of patients and interpretation of data. PS supervised the whole investigation and revised the manuscript. All authors read and approved the final manuscript.
\end{abstract}

\section{Acknowledgements}

This investigation was supported by Ministry of Health (Progetto Finalizzato) and INAIL to AP.

\footnotetext{
Author details

'Department of Neuroscience, University of Rome 'Tor Vergata', Rome, Italy. 'Intensive Care Unit, University of Rome 'Tor Vergata', Rome, Italy.

${ }^{3}$ Laboratory of Neuroimmunology, IRCCS, National Neurological Institute C. Mondino, Pavia, Italy. ${ }^{4}$ Laboratory of Neurophysiology, Santa Lucia Foundation, IRCCS, Rome, Italy.
}

Received: 8 May 2012 Accepted: 16 July 2012

Published: 8 August 2012

\section{References}

1. Weiss N, Miller F, Cazaubon S, Couraud PO: The blood-brain barrier in brain homeostasis and neurological diseases. Biochim Biophys Acta 2009, 1788:842-857.

2. Hirsch EC, Hunot S: Neuroinflammation in Parkinson's disease: a target for neuroprotection? Lancet Neurol 2009, 8:382-397.

3. Kortekaas R, Leenders KL, van Oostrom JC, Vaalburg W, Bart J, Willemsen AT, Hendrikse $\mathrm{NH}$ : Blood-brain barrier dysfunction in parkinsonian midbrain in vivo. Ann Neurol 2005, 57:176-179.

4. Carvey PM, Zhao CH, Hendey B, Lum H, Trachtenberg J, Desai BS, Snyder J, Zhu YG, Ling ZD: 6-Hydroxydopamine-induced alterations in blood-brain barrier permeability. Eur J Neurosci 2005, 22:1158-1168.

5. Westin JE, Lindgren HS, Gardi J, Nyengaard JR, Brundin P, Mohapel P, Cenci MA: Endothelial proliferation and increased blood-brain barrier permeability in the basal ganglia in a rat model of 3,4-dihydroxyphenylL-alanine-induced dyskinesia. J Neurosci 2006, 26:9448-9461.

6. Thompson EJ: Cerebrospinal fluid. J Neurol Neurosurg Psychiatry 1995, 59:349-357.

7. Teunissen CE, Petzold A, Bennett JL, Berven FS, Brundin L, Comabella M, Franciotta D, Frederiksen JL, Fleming JO, Furlan R, Hintzen RQ, Hughes SG, Johnson MH, Krasulova E, Kuhle J, Magnone MC, Rajda C, Rejdak K, Schmidt HK, van Pesch V, Waubant E, Wolf C, Giovannoni G, Hemmer B, Tumani H, Deisenhammer F: A consensus protocol for the standardization of cerebrospinal fluid collection and biobanking. Neurology 2009, 73:1914-1922.

8. Reiber H: Dynamics of brain-derived proteins in cerebrospinal fluid. Clin Chim Acta 2001, 310:173-186.

9. Gibb WR, Lees AJ: The significance of the Lewy body in the diagnosis of idiopathic Parkinson's disease. Neuropathol Appl Neurobiol 1989, 15:27-44.

10. Evans JR, Mason SL, Williams-Gray CH, Foltynie T, Brayne C, Robbins TW, Barker RA: The natural history of treated Parkinson's disease in an incident, community based cohort. J Neurol Neurosurg Psychiatry 2011, 82:1112-1118

11. Barbour R, Kling K, Anderson JP, Banducci K, Cole T, Diep L, Fox M, Goldstein JM, Soriano F, Seubert P, Chilcote TJ: Red blood cells are the major source of alpha-synuclein in blood. Neurodegener Dis 2008, 5:55-59.

12. McGeer PL, Itagaki S, Boyes BE, McGeer EG: Reactive microglia are positive for HLA-DR in the substantia nigra of Parkinson's and Alzheimer's disease brains. Neurology 1988, 38:1285-1291.

13. L'Episcopo F, Tirolo C, Testa N, Caniglia S, Morale MC, Cossetti C, D'Adamo P, Zardini E, Andreoni L, Ihekwaba AE, Serra PA, Franciotta D, Martino G, Pluchino S, Marchetti B: Reactive astrocytes and Wnt/ $\beta$-catenin signaling link nigrostriatal injury to repair in 1-methyl-4-phenyl-1,2,3,6-tetrahydropyridine model of Parkinson's disease. Neurobiol Dis 2011, 41:508-527.

14. Droździk M, Białecka M, Myśliwiec K, Honczarenko K, Stankiewicz J, Sych Z: Polymorphism in the P-glycoprotein drug transporter MDR1 gene: a possible link between environmental and genetic factors in Parkinson's disease. Pharmacogenetics 2003, 13:259-263.

15. Hawkins RA, Mokashi A, Simpson IA: An active transport system in the blood-brain barrier may reduce levodopa availability. Exp Neurol 2005 195:267-271.

16. Haussermann P, Kuhn W, Przuntek H, Muller T: Integrity of the bloodcerebrospinal fluid barrier in early Parkinson's disease. Neurosci Lett 2001, 300:182-184.

17. Fisone G, Snyder GL, Aperia A, Greengard P: Na+, K(+)-ATPase phosphorylation in the choroid plexus: synergistic regulation by serotonin/protein kinase $\mathrm{C}$ and isoproterenol/cAMP-PK/PP-1 pathways. Mol Med 1998, 4:258-265.

18. Silverberg GD, Huhn S, Jaffe RA, Chang SD, Saul T, Heit G, Von Essen A, Rubenstein E: Downregulation of cerebrospinal fluid production in patients with chronic hydrocephalus. J Neurosurg 2002, 97:1271-1275.

19. Woitalla D, Goetze O, Kim Jl, Nikodem AB, Schmidt WE, Przuntek H, Muller T: Levodopa availability improves with progression of Parkinson's disease. $J$ Neurol 2006, 253:1221-1226.

doi:10.1186/1742-2094-9-188

Cite this article as: Pisani et al:: Increased blood-cerebrospinal fluid transfer of albumin in advanced Parkinson's disease. Journal of Neuroinflammation 2012 9:188. 